\title{
Dilepton signals in heavy ion collisions at the CERN SPS
}

\author{
Carlos Lourenço ${ }^{\mathrm{a}}$
}

${ }^{\mathrm{a} C E R N}$, EP Division

CH-1211 Geneva 23, Switzerland

This paper overviews the main results on dilepton and heavy flavour production in heavy ion collisions, obtained in measurements done at the CERN SPS, over the last few years. The presently available results are very interesting, indicating the production of a new state of matter in central $\mathrm{Pb}-\mathrm{Pb}$ collisions at SPS energies. However, important questions remain open. New and improved measurements are needed to confirm beyond reasonable doubt the formation of a deconfined state of matter in heavy ion collisions at the SPS. The future data of a recently proposed experiment, to be carried out in the SPS in the years 2001-2003, should provide a much deeper understanding of the "new state of matter" hypothetically found at CERN.

\section{INTRODUCTION}

High energy heavy ion collisions is presently a major field in particle physics, with very active experimental programs at CERN (SPS) and at BNL (AGS), since 1986. With the RHIC collider in operation at BNL since this year and with a LHC experiment, ALICE, devoted to the study of heavy ion collisions, from 2005 onwards, it is clear that this field will remain very exciting during the next decade.

The main goal of this experimental program is the study of the phase diagram of strongly interacting matter, the discovery of the phase transition from confined hadronic matter to deconfined partonic matter, predicted by Lattice QCD calculations to occur when the system exceeds a given critical threshold in energy density or temperature, and the study of the thermo-dynamical properties of the new phase. The proof of existence of this phase and the study of its properties are key issues in the understanding of confinement and chiral-symmetry.

Once the existence of this new state of matter was proposed, some signatures of its formation in high energy heavy ion collisions were derived by theoretical arguments, among which we can emphasize the suppression of charmonia states $\left(\mathrm{J} / \psi, \chi_{c}\right.$ and $\left.\psi^{\prime}\right)$, due to the screening of the $c \bar{c}$ binding potential in the QGP colour soup, and the production of thermal dileptons, electromagnetic radiation emitted by the free quarks.

The results collected at the SPS were summarized in a special seminar held at CERN on February 2000, which was the basis of a "New State of Matter created at CERN" Press Release. Indeed, the SPS experiments have shown strong evidence that a new state of matter is produced in central $\mathrm{Pb}-\mathrm{Pb}$ collisions at the highest energies. Among the most exciting observations are the centrality dependence of the $\mathrm{J} / \psi$ suppression pattern 
measured by NA50, the low mass dilepton enhancement observed by CERES and the enhancement of dimuon production observed by NA38/NA50 and HELIOS-3.

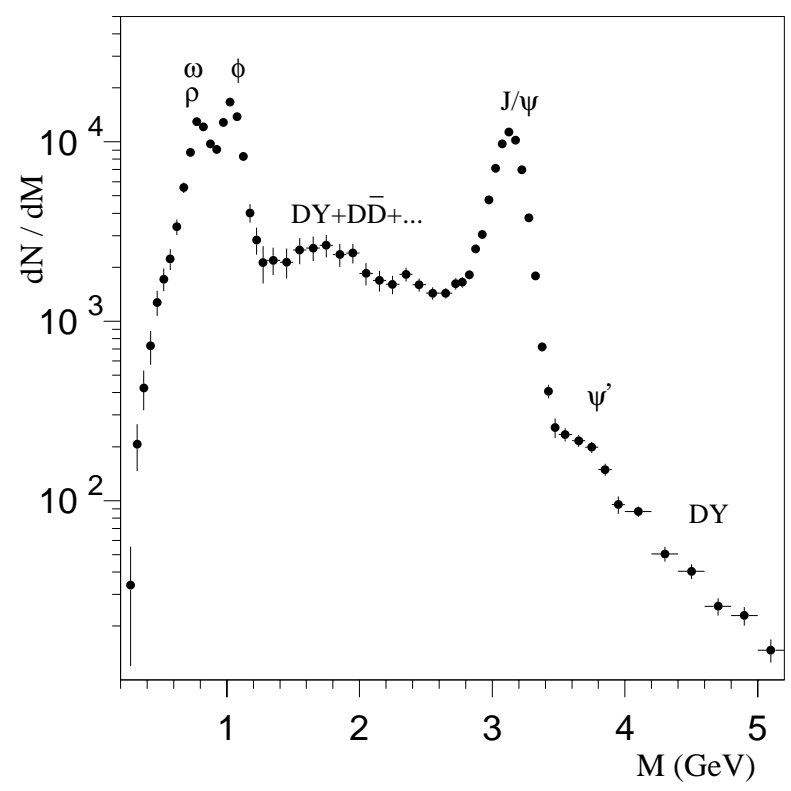

Figure 1. Mass distribution of dimuons produced in $\mathrm{Pb}-\mathrm{Pb}$ collisions, as measured by the NA50 experiment.

Figure 1 shows a typical dimuon mass distribution produced in heavy ion collisions. Such measurements provide information on low and intermediate mass dilepton production, and allow to study the production of the $\rho, \omega, \phi, \mathrm{J} / \psi$ and $\psi^{\prime}$ resonances.

\section{RECENT RESULTS}

\subsection{Low mass dileptons}

The CERES experiment has observed [1] that the yield of low mass $e^{+} e^{-}$pairs measured in $\mathrm{p}$-Be and $\mathrm{p}-\mathrm{Au}$ collisions is properly described by the expected "cocktail" of hadronic decays. In Pb-Au collisions, on the contrary, the measured yield [2] exceeds by a factor of 2.5 the expected signal in the mass region $0.2-0.7 \mathrm{GeV}$, as shown in Fig. 2. Dileptons from $\pi^{+} \pi^{-}$annihilation would increase the expected yield around the mass of the $\rho$ meson, not reproducing the measured shape. The excess dileptons are concentrated at low $p_{\mathrm{T}}$ (Fig. 3) and their yield seems to scale with the square of the charged particle multiplicity (Fig. 4).

These observations are consistent with the expectation that the properties of the vector mesons should change when produced in dense matter, due to medium effects. In particular, near the phase transition to the quark-gluon phase, chiral symmetry should be partially restored. As a consequence, vector mesons should become indistinguishable from their chiral partners, inducing changes in the masses and decay widths of the mesons [3]. The short lifetime of the $\rho$ meson, shorter than the expected lifetime of the dense system produced in the SPS heavy ion collisions, makes it a sensitive probe of medium effects and, in particular, of chiral symmetry restoration. 

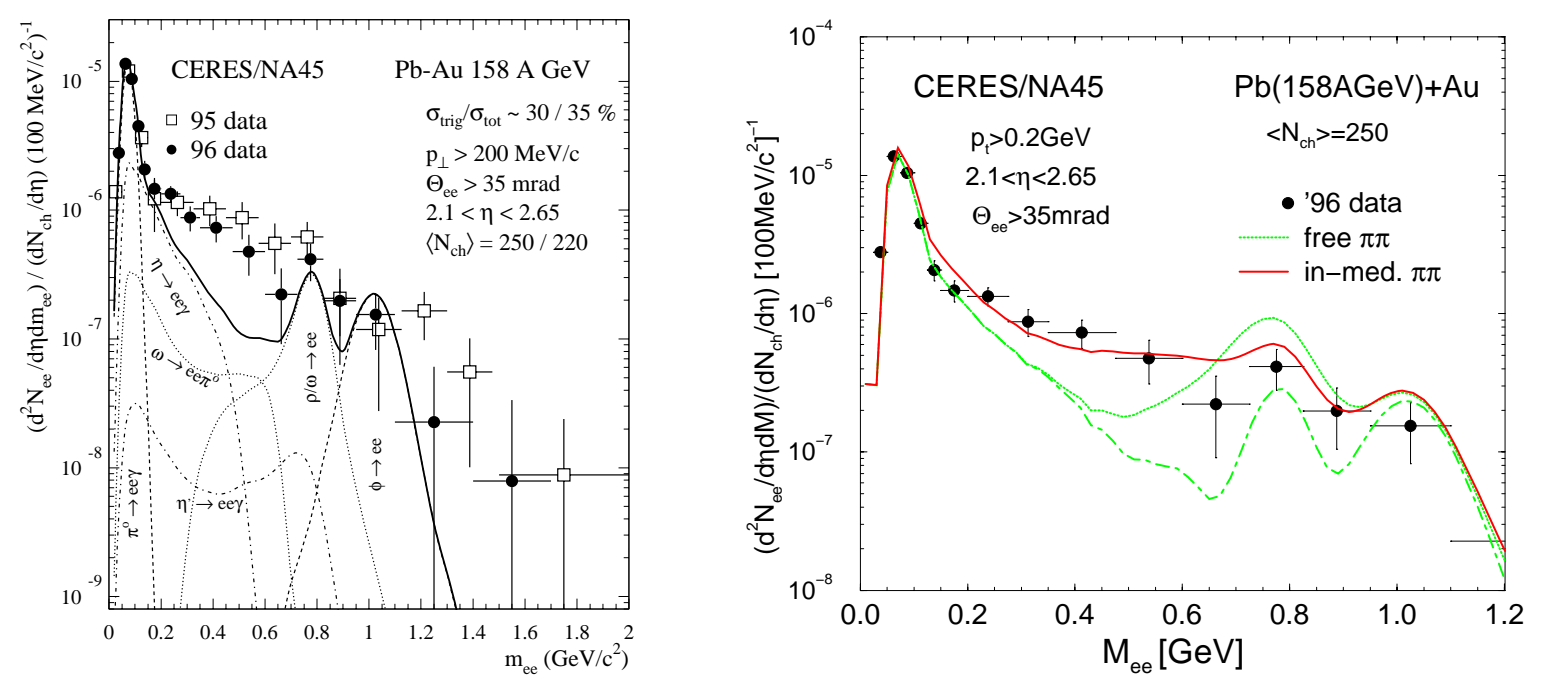

Figure 2. Dielectron mass distribution measured by CERES in Pb-Au collisions, compared to the expected hadronic decays (left) and to the contribution from $\pi \pi$ annihilation with and without in medium effects (right).

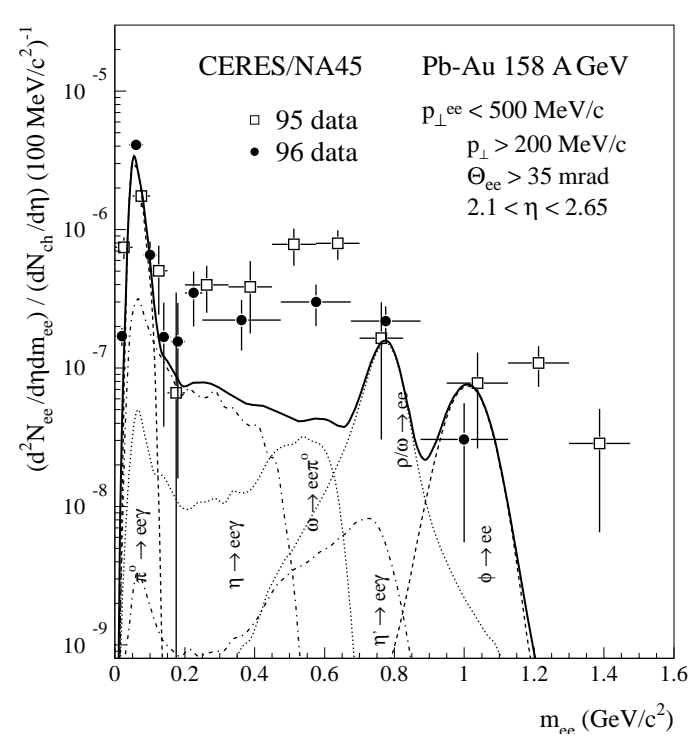

Figure 3. Mass distribution of low $p_{\mathrm{T}}$ dielectrons produced in $\mathrm{Pb}-\mathrm{Au}$ collisions, as measured by CERES.

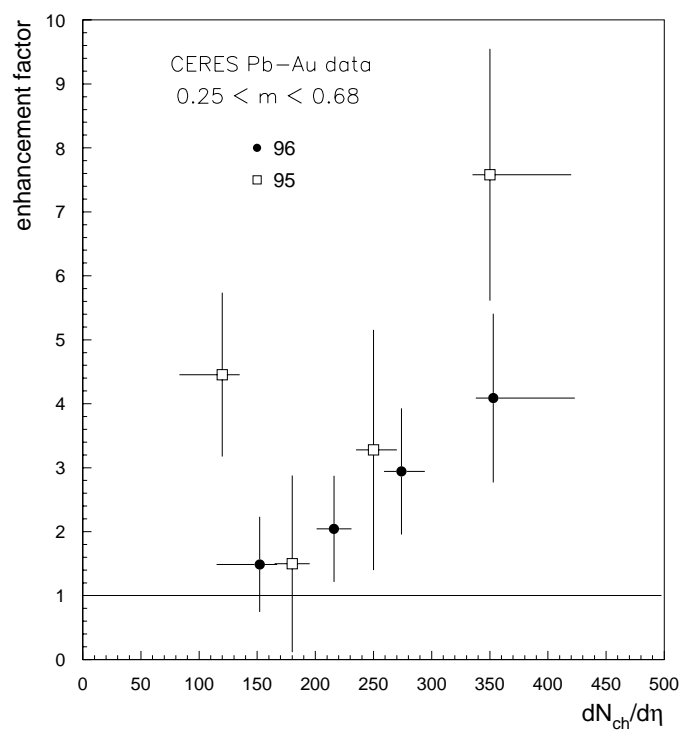

Figure 4. Yield of excess dileptons as a function of the charged particle multiplicity. Is there an onset at $\mathrm{d} N_{\mathrm{ch}} / \mathrm{d} \eta \sim 100$ ? 
The present measurements are not accurate enough to clearly distinguish between a change in the $\rho$ mass (signaling the restoration of chiral symmetry) and a broadening due to conventional hadronic interactions [4]. CERES has recently added a TPC to the experiment [5], to improve the momentum resolution of the dielectron measurement. Given enough collected statistics, the new data should clarify the shape of the signal mass distribution in the resonance $(\rho, \omega)$ region, and rule out some of the present interpretations.

\subsection{Intermediate mass dimuons}

The NA38 and NA50 experiments have studied [6] the production of dileptons in the mass window between the $\phi$ and the $\mathrm{J} / \psi$, as a superposition of Drell-Yan dimuons and simultaneous semileptonic decays of $D$ and $\bar{D}$ mesons, after subtraction of the combinatorial background from pion and kaon decays.

The Drell-Yan and open charm contributions were calculated with the PYTHIA event generator [7] with the MRS A set of parton distribution functions [8]. PYTHIA describes reasonably well [9] the kinematics and cross sections (including the energy dependence) of $D$ meson hadroproduction, as well as the semi-leptonic decays and the corresponding lepton distributions. Figure 5 shows that the dimuon mass spectra measured by NA50 in p-A collisions are very well reproduced taking the high mass region (above $4.5 \mathrm{GeV}$ ) to normalize the Drell-Yan component and an open charm cross-section in good agreement with direct measurements of other experiments. The calculations do not include NLO QCD diagrams, particularly important for high $p_{\mathrm{T}}$ Drell-Yan production.
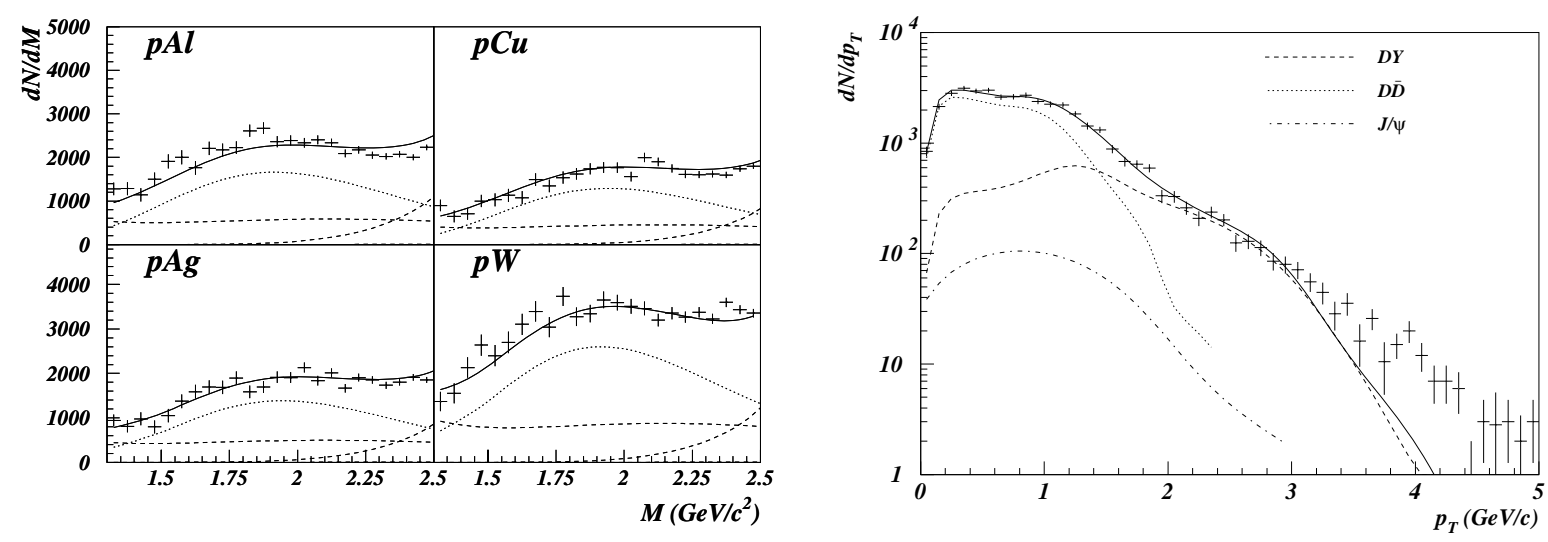

Figure 5. Dimuon mass (left) and $p_{\mathrm{T}}$ (right) distributions measured in p-A collisions by NA50, compared to the corresponding expected sources.

The superposition of Drell-Yan and open charm contributions, with the nucleon-nucleon absolute cross sections scaled with the product of the mass numbers of the projectile and target nuclei (as expected for hard processes), fails to properly describe the intermediate mass dimuons measured in ion collisions. Figure 6 shows, for central $\mathrm{Pb}-\mathrm{Pb}$ collisions, how the sum of the expected sources underestimates the measured data. The same figure shows that the data can be reproduced by simply increasing the open charm yield. The scaling factor by which the charm contribution should be multiplied to properly describe 
the measured spectra seems to grow linearly with the number of nucleons participating in the collision, as shown in Fig. 7. In this figure, the points "4-D analysis" are obtained with an improved deconvolution method to extract the physical kinematics from the measured values, affected by acceptance and finite resolution (smearing) effects. This analysis method accounts for physical correlations among kinematical variables and does not require any assumption on the specific shapes of their distributions [10].

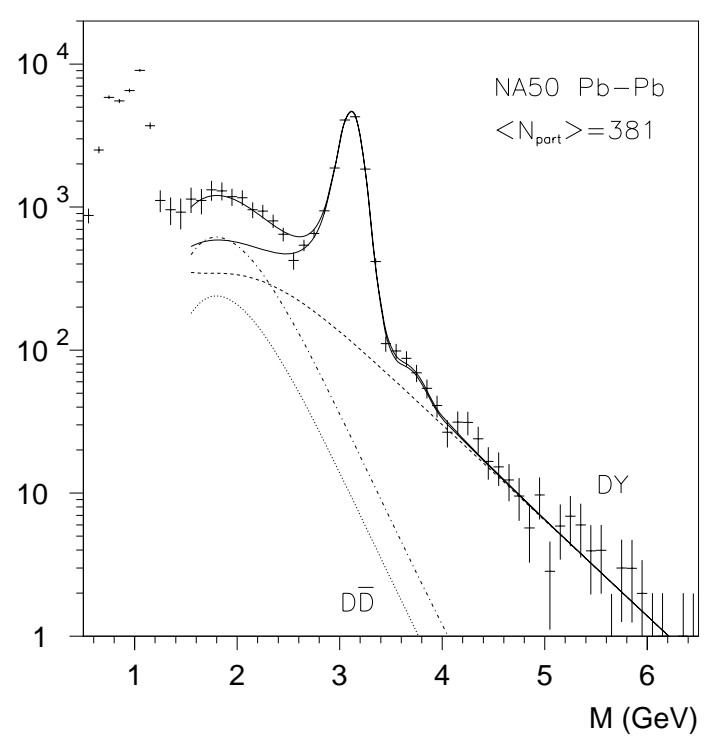

Figure 6. Dimuon mass distribution measured in central $\mathrm{Pb}-\mathrm{Pb}$ collisions, compared to the expected sources, with and without scaling up the charm contribution.

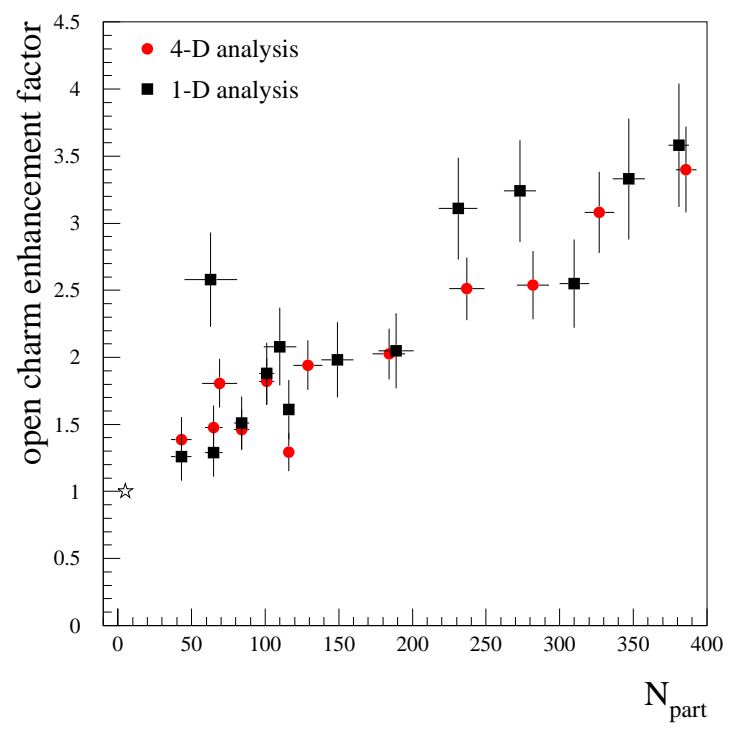

Figure 7. Dependence on the number of participants of the scaling factor by which the charm contribution must be multiplied to properly describe the measured spectra.

The observed excess can be due to an enhancement of open charm production in heavy ion collisions [11]. The rescattering of the charm quarks or $D$ mesons in the produced medium could lead to a broader $p_{\mathrm{T}}$ distribution and locally enhance the charm component in the limited phase space domain covered by NA50 [12], but recent studies [13] have shown that the data cannot be accounted for by this model. The excess can also be due to the production of thermal dimuons, an old signal recently revisited [14,15]. In particular, the intermediate mass dimuons produced in the most central $\mathrm{Pb}-\mathrm{Pb}$ collisions are well reproduced [13] by adding thermal radiation, calculated according to Ref. [14], to the Drell-Yan and charm contributions normally extrapolated from nucleon-nucleon collisions. Note that this model explicitly includes a QGP phase transition at a critical temperature of $175 \mathrm{MeV}$. The present data cannot distinguish between an absolute enhancement of charm production and the emission of thermal dilepton radiation.

\subsection{Charmonia production and suppression}

The formation of a deconfined medium should induce a considerable suppression of the charmonia production rate, due to the colour 'Debye' screening of the $c \bar{c}$ potential 
or to the breaking of the $c \bar{c}$ bound by scattering with energetic (deconfined) gluons [16]. However, even the relatively simple measurement of $\mathrm{J} / \psi$ production faces a big challenge when it comes to furnish a convincing logical case that proves, to the satisfaction of the experts in the field, that a deconfined state of matter has been formed. It is not enough to show that a certain observable changes from $\mathrm{p}-\mathrm{Pb}$ to $\mathrm{Pb}-\mathrm{Pb}$ collisions, for instance, or to argue that its value in the most central nucleus-nucleus collisions is different from what is calculated in a "conventional physics" model.

The best path to clearly establish a solid result and shed light in this complicated field is to build a robust set of measurements, that establishes a precise reference baseline, relative to which the specific behaviour of heavy ion collisions can be extracted. Such a baseline shows what is the "normal" behaviour of the signal we are studying, before we start looking for changes due to QGP formation. Furthermore, we are in a much better position if nature provides us with a reference process, insensitive to the formation of a deconfined phase, specially if we can measure it with the same detector.

In the case of the $\mathrm{J} / \psi$ suppression topic, the baseline is built from the measurements done with pp, p-A and light ion collisions. The best reference physics process, at SPS energies, is the rate of high mass dilepton production, since the Drell-Yan process can be precisely calculated and depends on the collision system in a well known way. Figure 8 shows absolute cross sections of Drell-Yan production measured by the NA38, NA51 and NA50 experiments in pp, p-D, p-W, S-U and Pb-Pb collisions, at several energies, divided by the corresponding values calculated at leading order with the MRS A parton distribution functions.

From the measured $\mathrm{J} / \psi$ production yields we can derive the $\mathrm{J} / \psi$ cross section per nucleon, $B_{\mu \mu}^{\psi} \sigma^{\psi} / \mathrm{AB}$, displayed in Fig. 9 as a function of the product of the mass numbers

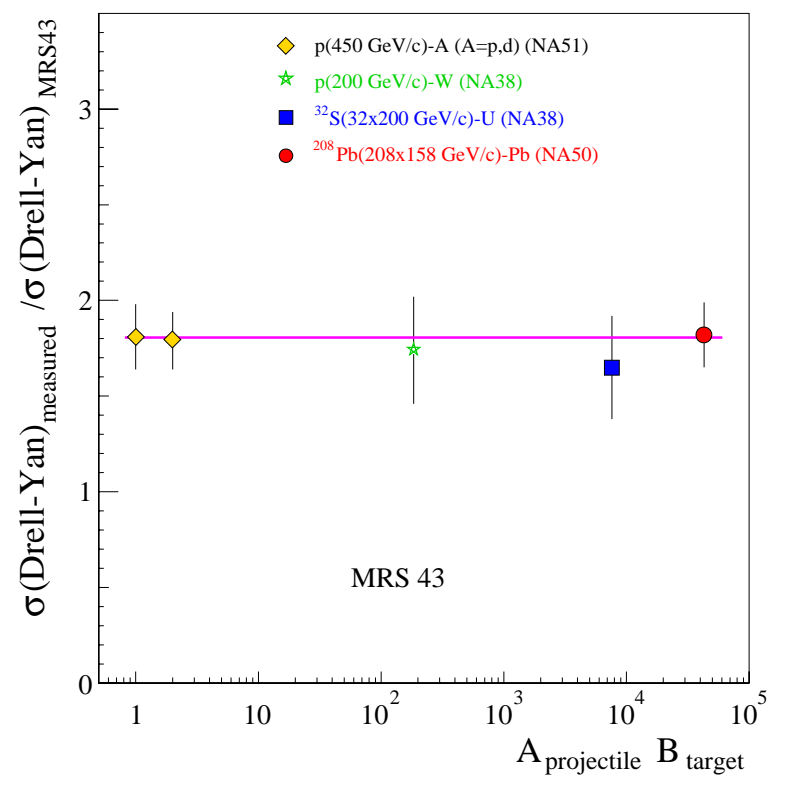

Figure 8. The measured yield of Drell-Yan dimuons follows the expected values, from $\mathrm{pp}$ to $\mathrm{Pb}-\mathrm{Pb}$ collisions.

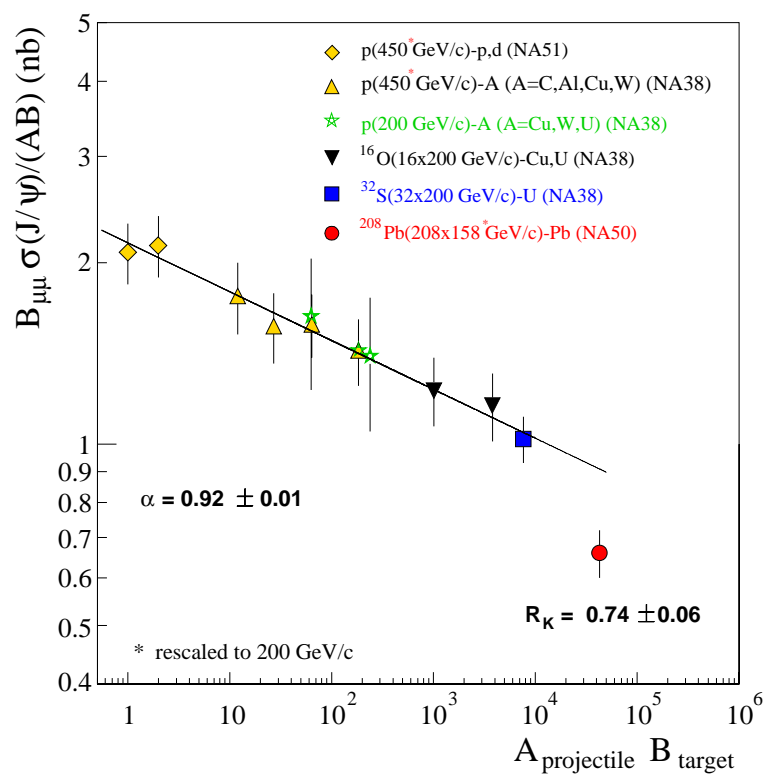

Figure 9. The $\mathrm{J} / \psi$ production cross section measured from pp to $\mathrm{Pb}-\mathrm{Pb}$ collisions, in the NA38, NA51 and NA50 experiments. 
of the two colliding nuclei. Contrary to what happens with the evolution of the DrellYan process, the $\mathrm{Pb}-\mathrm{Pb} \mathrm{J} / \psi$ point is completely different from the value indicated by the pattern established by the p-A and light ion measurements.

The peculiar behaviour of the $\mathrm{Pb}-\mathrm{Pb}$ data can be seen in much more detail by binning the collected event sample as a function of the centrality of the collisions. Figure 10 shows how the $\mathrm{J} / \psi$ production rate, with respect to the yield of Drell-Yan dimuons, decreases from peripheral to central $\mathrm{Pb}-\mathrm{Pb}$ collisions, using either $E_{\mathrm{ZDC}}$ or $E_{\mathrm{T}}$ to estimate the centrality of the reactions. The same figures show the "normal $\mathrm{J} / \psi$ absorption line", determined by the reference data (from pp to S-U) shown in Fig. 9. The data points collected in the most central collisions show a very significant departure from the expected behaviour, while the most peripheral points seem to be in (rough) agreement with the absorption expected in normal nuclear matter.
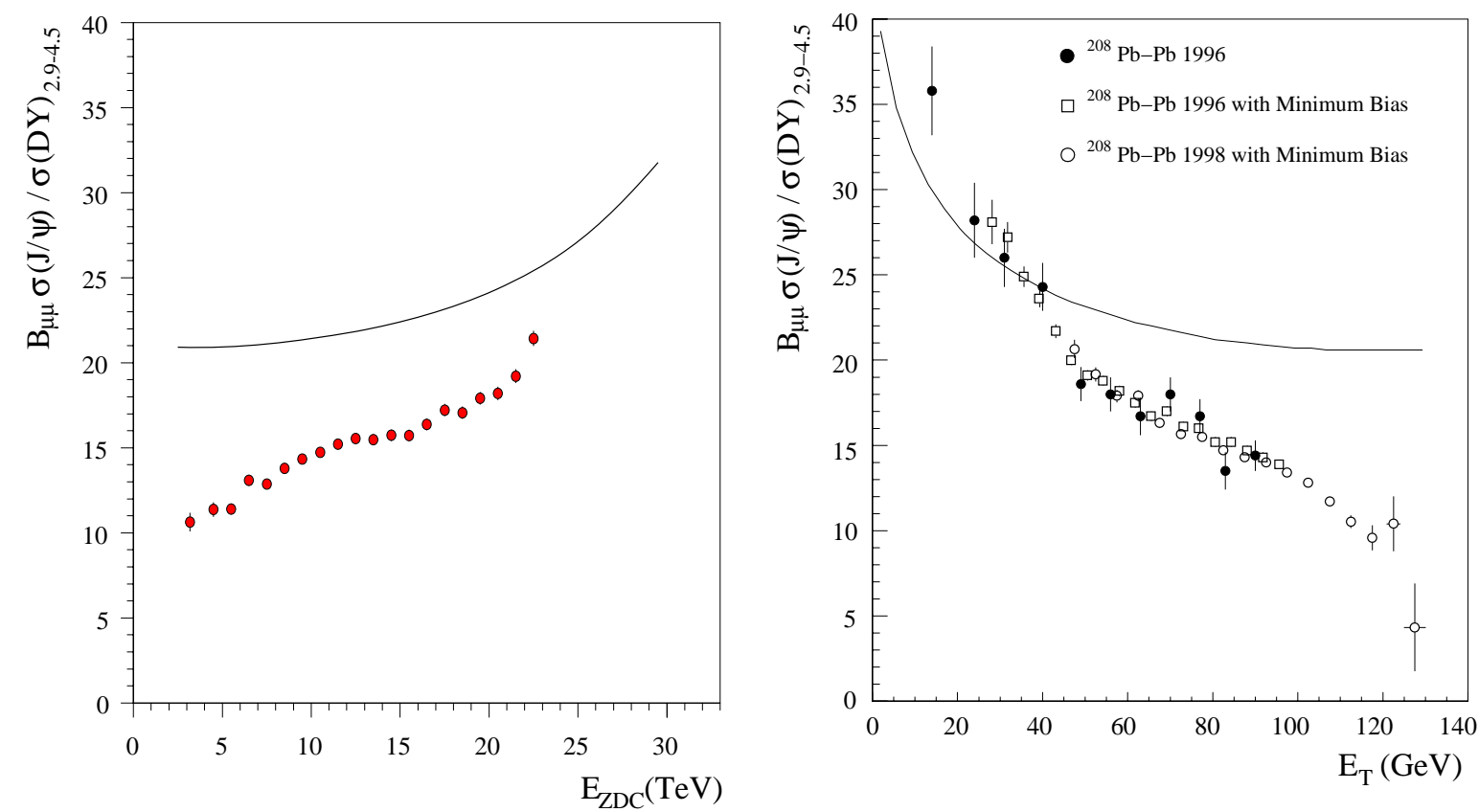

Figure 10. The $\mathrm{J} / \psi$ suppression pattern as a function of the forward (left) and transverse (right) energy, in $\mathrm{Pb}-\mathrm{Pb}$ collisions, as measured by NA50.

The left panel of Fig. 11 shows that the observed suppression pattern is in clear disagreement with the predictions of the "conventional" models [17-20], that attribute the disappearance of the $\mathrm{J} / \psi$ mesons to interactions with "comoving" hadrons. A first departure from the conventional curves is seen for collisions that release around $40 \mathrm{GeV}$ of neutral transverse energy in the electromagnetic calorimeter of NA50. A second substantial drop is seen for the most central $\mathrm{Pb}-\mathrm{Pb}$ collisions, while the hadronic models systematically predict a smooth absorption trend. On the other hand, a two-step pattern, as seen in the data, is expected in case the charmonia states are dissolved in a deconfined medium, due to the different melting temperatures of the $\chi_{c}$ and $\mathrm{J} / \psi$ states. The addition of the pp, p-A and S-U results makes the right panel of Fig. 11 particularly illustrative of the unconventional nature of the observed $\mathrm{J} / \psi$ suppression pattern. 

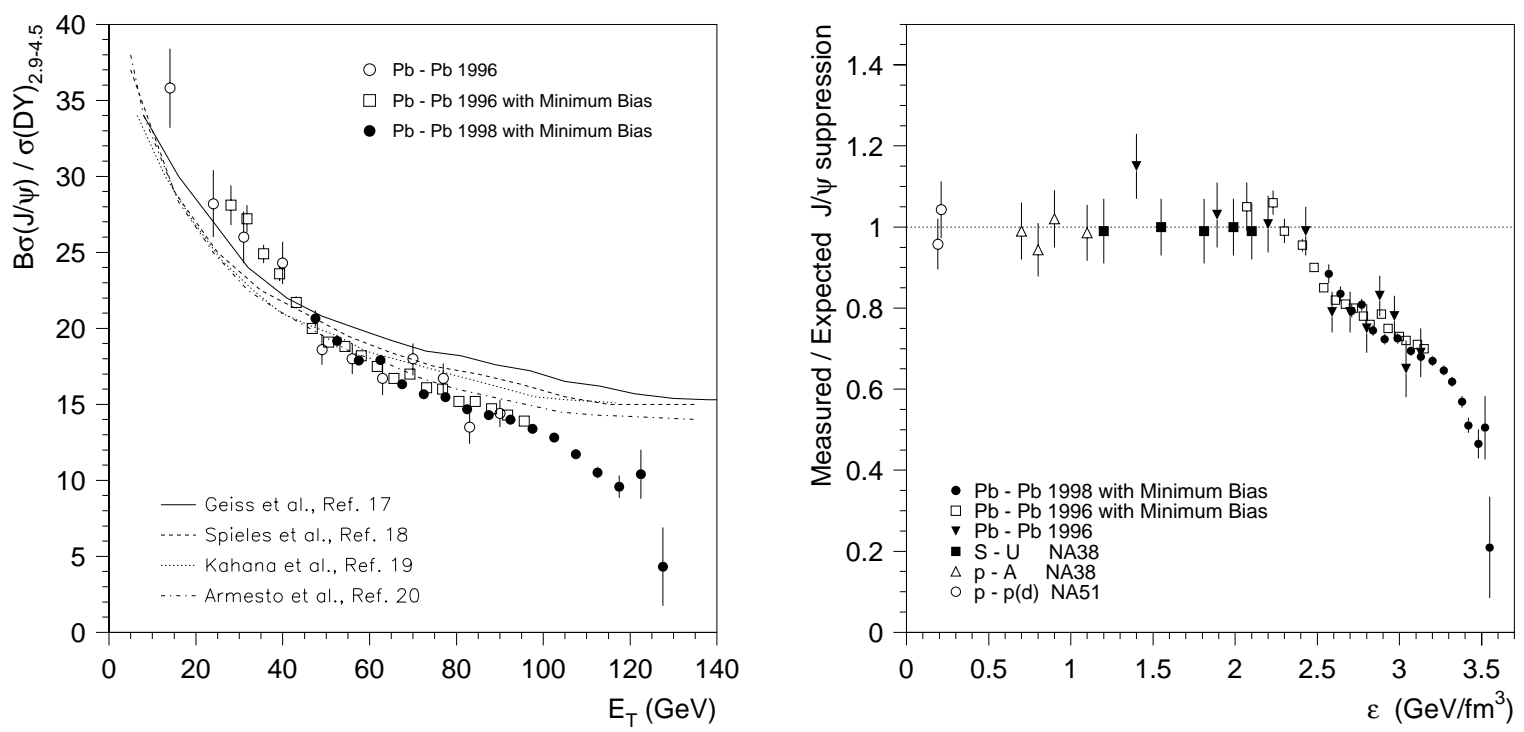

Figure 11. The measured $\mathrm{J} / \psi$ suppression pattern rules out the presently available conventional models (left) and is in qualitative agreement with the two-step pattern expected in the deconfinement picture (right).

\section{OPEN QUESTIONS}

The current interpretation of the step-wise pattern of $\mathrm{J} / \psi$ suppression measured by NA50 in $\mathrm{Pb}-\mathrm{Pb}$ collisions is that there is a first drop when the (local) energy density is above the threshold for melting the $\chi_{c}$ charmonia states, and a second drop when the more tightly bound $\mathrm{J} / \psi$ state itself starts melting. If we accept that this pattern indicates the production of a state of matter where colour is no longer confined, we must move on to the detailed understanding of how deconfinement sets in, and what physics variable governs the threshold behaviour of charmonia $\left(\chi_{c}\right)$ suppression: (local) energy density, density of wounded nucleons, density of percolation clusters, etc. This requires collecting data with smaller nuclear systems like In-In. Indeed, it is possible to predict at which impact parameter, $b$, of In-In collisions is reached the same threshold in (local) energy density, or any other variable, as reached in $\mathrm{Pb}-\mathrm{Pb}$ collisions of $b \approx 8 \mathrm{fm}$, where the $\chi_{c}$ state starts melting. According to the deconfinement model used in the calculation [21] shown in Fig. 12, the onset of anomalous $\mathrm{J} / \psi$ suppression should happen at an impact parameter $b=2.0-2.5 \mathrm{fm}$, in In-In collisions at $158 \mathrm{~A} \mathrm{GeV}$. A verification of this prediction would be the final element of proof that the deconfined quark-gluon phase sets in, and would provide fundamental information on the mechanisms behind the observed phenomena.

The $\mathrm{J} / \psi$ data do not provide a direct measurement of the critical temperature. Finite temperature lattice QCD tells us that the strongly bound $\mathrm{J} / \psi c \bar{c}$ state should be screened when the medium reaches temperatures 30-40\% higher than $T_{c}$, while the larger and more loosely bound $\psi^{\prime}$ state should melt near $T_{c}$. The $\psi^{\prime}$ is already significantly suppressed when going from $\mathrm{p}-\mathrm{U}$ to peripheral $\mathrm{S}-\mathrm{U}$ collisions but the presently existing results are not clear in what concerns the onset and pattern of the $\psi^{\prime}$ suppression. Figure 13 shows the ratio between the $\psi^{\prime}$ and Drell-Yan production rates, as a function of L, the thickness of 

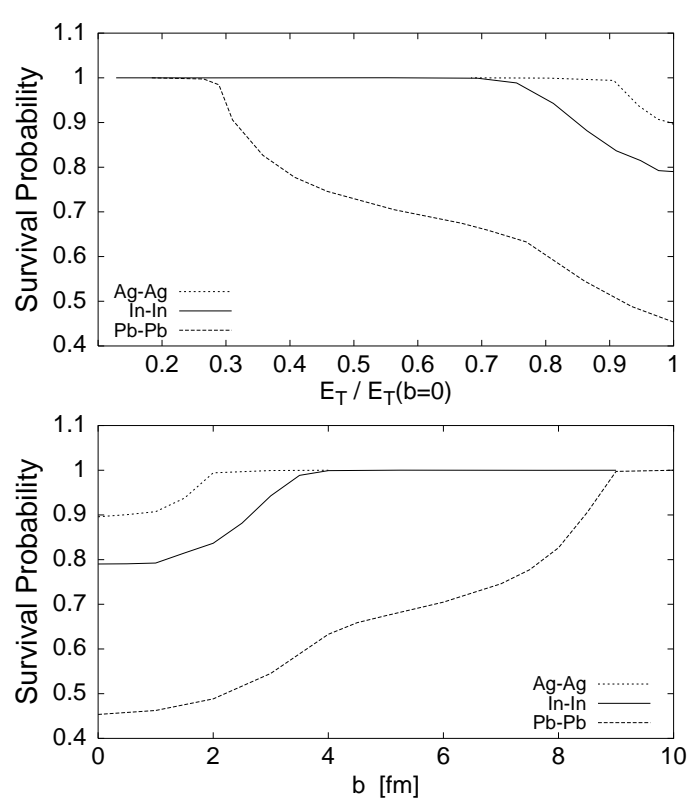

Figure 12. The $\mathrm{J} / \psi$ suppression pattern predicted in a deconfinement model for different collision systems.

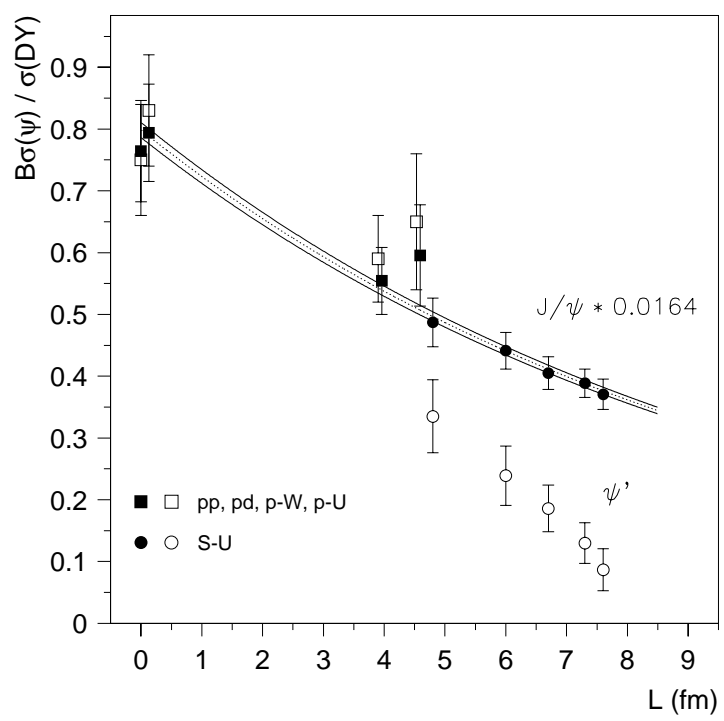

Figure 13. The $\psi^{\prime}$ yield (open symbols) is strongly suppressed in $\mathrm{S}-\mathrm{U}$ collisions. Hadronic absorption or Debye screening?

nuclear matter crossed by the charmonia states. The corresponding $\mathrm{J} / \psi$ (normal nuclear absorption) pattern is also shown, scaled down by the factor $1.64 \%$. Is the "anomalous" $\psi^{\prime}$ suppression due to melting or to hadronic absorption? If we see that this suppression happens abruptly, within a single collision system rather than comparing $\mathrm{p}-\mathrm{U}$ to $\mathrm{S}-\mathrm{U}$ data, we would know that Debye screening is the mechanism responsible for the $\psi^{\prime}$ suppression and we would have a clear measurement of $T_{c}$. That requires a new measurement, with improved mass resolution to have a cleaner separation of the $\psi^{\prime}$ peak with respect to the $\mathrm{J} / \psi$, and which scans the energy density region from the $\mathrm{p}-\mathrm{U}$ to the $\mathrm{S}-\mathrm{U}$ data. In-In collisions are also well placed for this study.

It is also very important to have a much better understanding of the nuclear dependence of the $\chi_{c}$ production cross section, in p-A collisions, at SPS energies, since the radiative decay of the $\chi_{c}$ states leads to $30-40 \%$ of the $\mathrm{J} / \psi$ 's measured in proton induced collisions.

Knowing that the bound $c \bar{c}$ states are suppressed, it is natural to ask what happens to the unbound charm. Charm quarks are so heavy that they can only be produced at the earlier stages of the nuclear collision, before the eventual formation of the QGP state. Charm is the heaviest flavour that can be studied in heavy ion collisions at the SPS energies. The production of charm quarks leads mainly to correlated pairs of $D$ and $\bar{D}$ mesons. Only a few percent of the charmed quark pairs end up in the bound charmonia states presently studied by the NA50 experiment, and which exhibit a rather interesting "anomalous" behaviour. What happens to the vast majority of $c$ quarks? Are they affected by energy loss while crossing the dense (partonic or hadronic) medium? Is charm production enhanced similarly to what has been seen in the strangeness sector? $D$ meson production also provides the natural reference with respect to which we should 
study the observed $\mathrm{J} / \psi$ suppression, since both production mechanisms depend on the same gluon distribution functions.

Measuring open charm production will also clarify if the enhancement of intermediate mass dimuons is due to the simultaneous semileptonic decays of $D$ mesons. If the origin of this excess is due to charm enhancement, it makes the $\mathrm{J} / \psi$ suppression even more anomalous. If, instead, it is due to thermal virtual photons from the QGP, it becomes the first direct observation of thermal radiation, providing a new electromagnetic probe through which we can investigate how hot dense matter is created. Thermal dileptons should be distinguishable from other sources of prompt dimuons through their mass and $p_{\mathrm{T}}$ distributions and through the characteristic dependence of their yield on the multiplicity of charged particles.

The understanding of low mass dilepton production also requires a more accurate measurement than presently available, in terms of statistics, mass resolution and signal to background ratio. In particular, data on the $\omega$ and $\phi$ peaks, down to zero transverse momentum and with a detailed centrality scan, will provide a good reference with respect to which the $\rho$ and "continuum" signals can be studied.

\section{FUTURE PROSPECTS}

The results and open questions presented in the previous sections emphasize the importance of having a direct measurement of open charm production in heavy ion collisions at SPS energies, besides significantly improving several other measurements. A dedicated experiment is needed, that can cope with the very high particle multiplicities reached in the most central ion collisions (more than 400 charged particles per unit rapidity at midrapidity) and with the rather small $D$ production cross section. The NA50 experiment already uses CERN's highest intensity heavy ion beam (more than $10^{7}$ ions per second) and has a very clean dimuon trigger, quite appropriate to look for rare processes. The NA60 experiment has recently been proposed [22], re-using the NA50 muon spectrometer and completely redesigning the target area, with the latest technology in terms of (radiation tolerant) high granularity trackers, in order to have access to the muon tracks at the vertex level and vastly improve the physics capability of the detector $[22,23]$. The following questions summarize the physics motivation of this experiment.

- Is the open charm yield enhanced in nucleus-nucleus collisions? How does it compare to the suppression pattern of bound charm states?

- What is the variable (local energy density, cluster density, etc.) that rules the onset of charmonia suppression?

- What is the origin of the $\psi^{\prime}$ suppression? If it is due to Debye screening, what is its melting temperature?

- What fraction of $\mathrm{J} / \psi$ come from $\chi_{c}$ decays? Does it change from $\mathrm{p}-\mathrm{Be}$ to $\mathrm{p}-\mathrm{Pb}$ collisions?

- What is the origin of the dimuon excess seen in the intermediate mass region? Changes in the $D$ meson cross section (or kinematics) or production of thermal dimuons?

- Are there medium induced modifications in the $\rho$ meson?

- Is the observed $\phi$ enhancement a specific feature of heavy ion collisions? 
The NA60 experiment is expected to take place at the CERN-SPS from 2001 to 2003, using proton and heavy ion beams. The NA60 detector complements the muon spectrometer and zero degree calorimeter already used in the NA50 experiment with two stateof-the-art silicon detectors, placed in the target region: a radiation hard beam tracker, based on a set of four silicon microstrip detectors placed on the ion beam and operated at $130 \mathrm{~K}$ (the "beamscope") and a silicon pixel telescope, made of 88 Alice1 pixel chips, of around 8000 channels each.

The beamscope gives the transverse coordinates of the impact of the incident ions on the targets with a precision of order $20 \mu \mathrm{m}$, allowing the identification of the interaction point with enough accuracy to measure the offset of the muon tracks and thereby select events where a pair of charmed mesons was produced in the collision. It should stand a radiation dose rate of around 1 Grad per day [24].

The 10-plane silicon pixel telescope, placed in a $1.7 \mathrm{~T}$ dipole field, is made with pixel readout chips developed using radiation-tolerant design techniques and in $0.25 \mu \mathrm{m}$ CMOS technology. Test beam results with prototype versions showed an excellent performance up to radiation doses of around $30 \mathrm{Mrad}$ [25]. This detector will be a natural test bench for the pixel layers of the Inner Tracker System of the ALICE experiment, which uses basically the same pixel detectors and readout electronics. The improvement in the mass resolution of the dimuon measurement when adding the silicon pixel telescope has been demonstrated in feasibility tests done in 1998, using the previous generation of pixel chips [26]. The results, shown in Fig. 14, confirm that the mass resolution improves from $70 \mathrm{MeV}$ to $20 \mathrm{MeV}$ at the $\omega$ mass, as expected from the Monte Carlo simulations illustrated on Fig. 15.
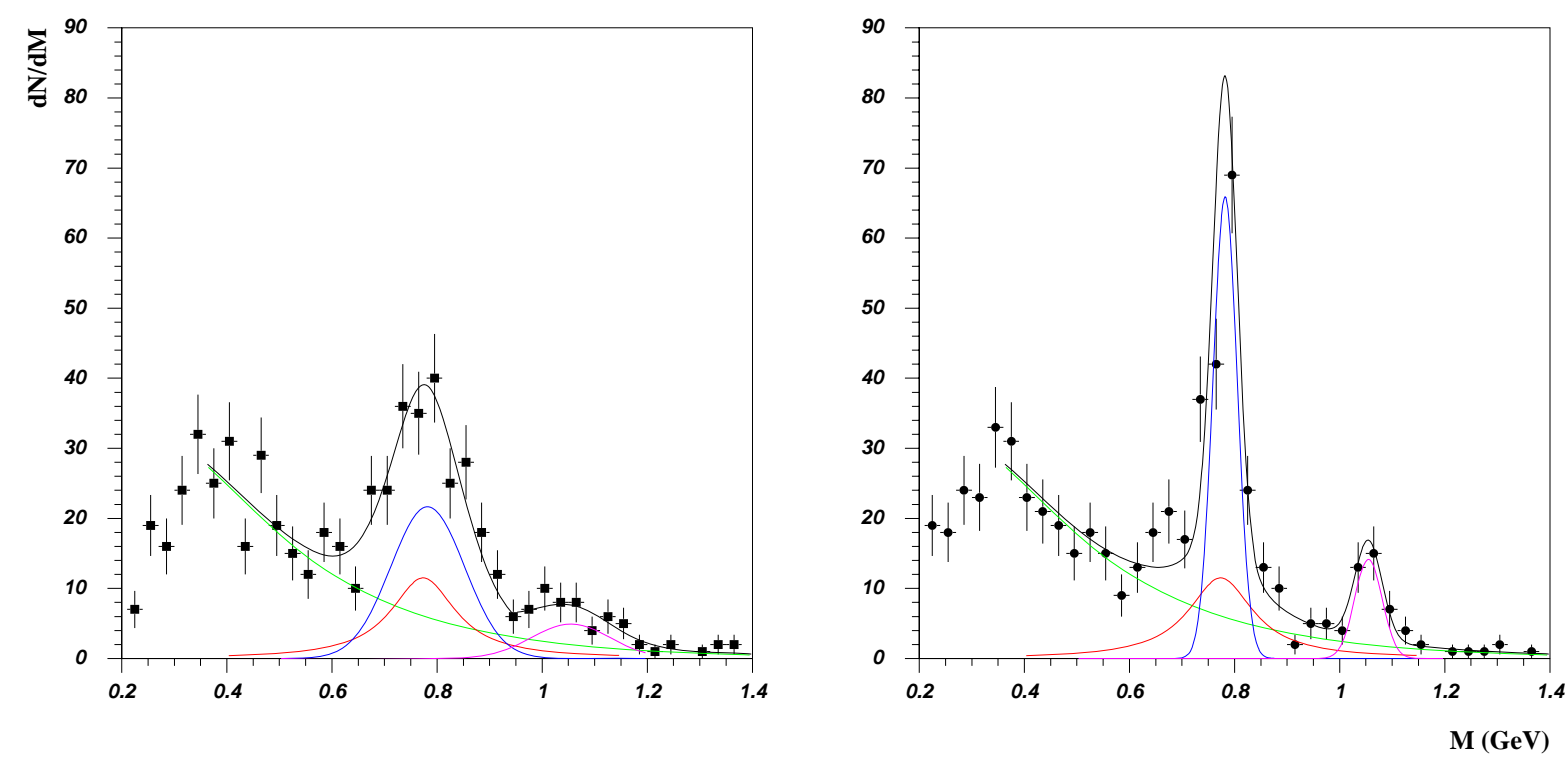

Figure 14. Dimuon mass distributions measured in 1998, in p-Be collisions, before (left) and after (right) using the test pixel telescope's information. The curves are normalized to the same number of events in both figures. 

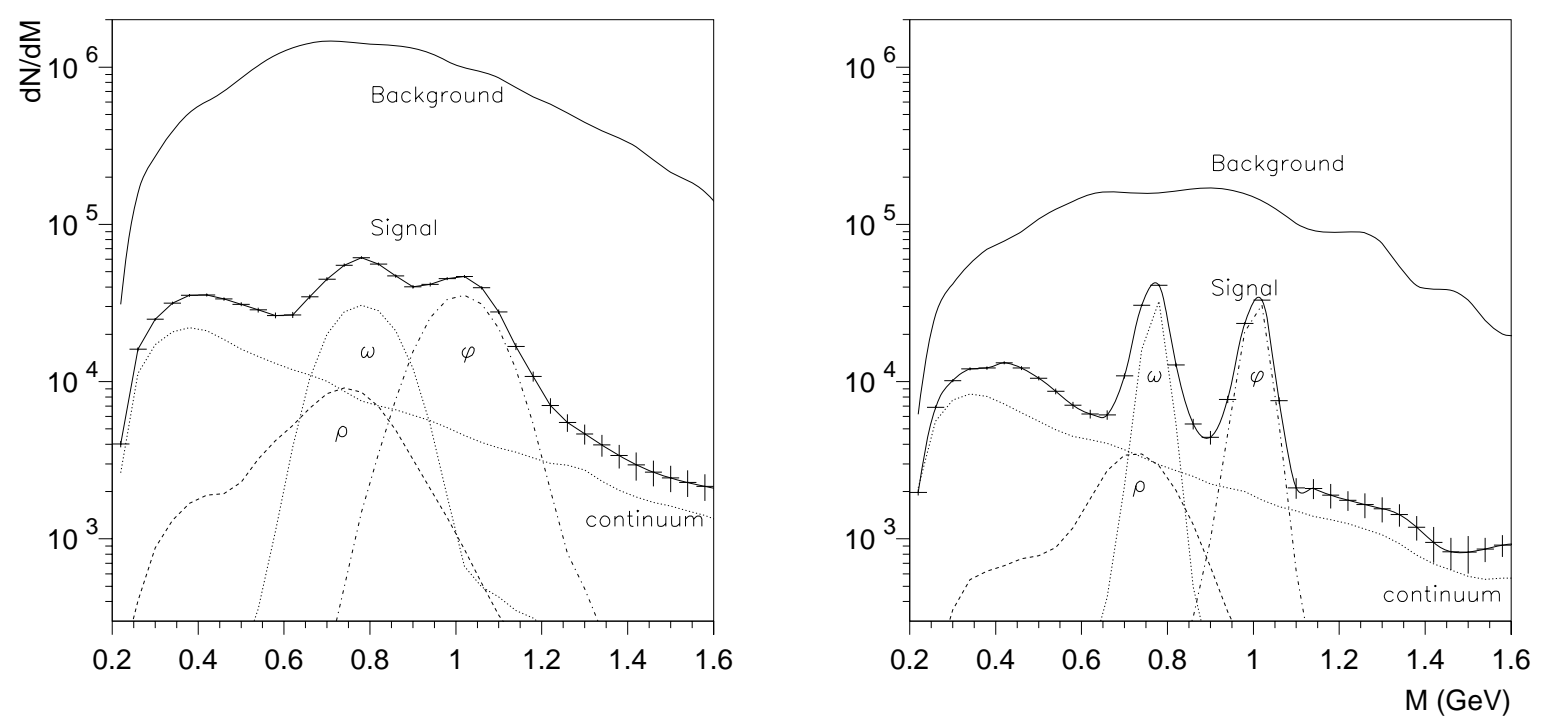

Figure 15. Simulated dimuon mass distribution in $\mathrm{Pb}-\mathrm{Pb}$ collisions, before (left) and after (right) using the NA60 pixel telescope information. The statistics corresponds to around one week of running.

By measuring the impact parameter of the muon tracks, i.e. the minimum distance between the track and the collision vertex, in the transverse plane, NA60 will be able to separately study the production of prompt dimuons and the production of muons originating from the decay of charmed mesons, in p-A and heavy ion collisions. The prompt dimuon analysis will use events where both muons come from (very close to) the interaction vertex. The open charm event sample is composed of those events where both muon tracks have a certain minimum offset with respect to the interaction point and a minimal distance between themselves at $z_{\text {vertex }}$. Figure 16 shows the simulated mass spectra for both event samples. It should not be very difficult to see which of these two event samples is enhanced by a factor of 2-3 in nuclear collisions of $N_{\text {part }} \sim 300$.

Figure 17 shows the accuracy of the determination of the interaction point, in the transverse plane. In the beam direction the vertex is found with a precision of $\sim 100-150 \mu \mathrm{m}$. If the incident nucleus makes a peripheral collision and the beam spectators fragment collides further down in the target, the double emission of particles should be distinguishable from what happens in a single (central) collision. This allows the use of a thicker target, with a corresponding gain in total effective luminosity. A high interaction rate is the basis for enough statistics to study the charmonia production yield in many centrality bins, a necessary condition to accurately determine a step-wise suppression pattern. Besides statistics, it is also important to have a good accuracy in the measurement of the centrality of the collision. Figure 18 shows that a resolution between $5 \%$ and $10 \%$, depending on the centrality, should be reached in NA60, using the forward energy, $E_{\mathrm{ZDC}}$, and the charged particle multiplicity, $\mathrm{d} N_{\mathrm{ch}} / \mathrm{d} y$.

The NA60 experiment will also study prompt dimuon and open charm production in p-A collisions. These are important reference measurements, to understand the results 

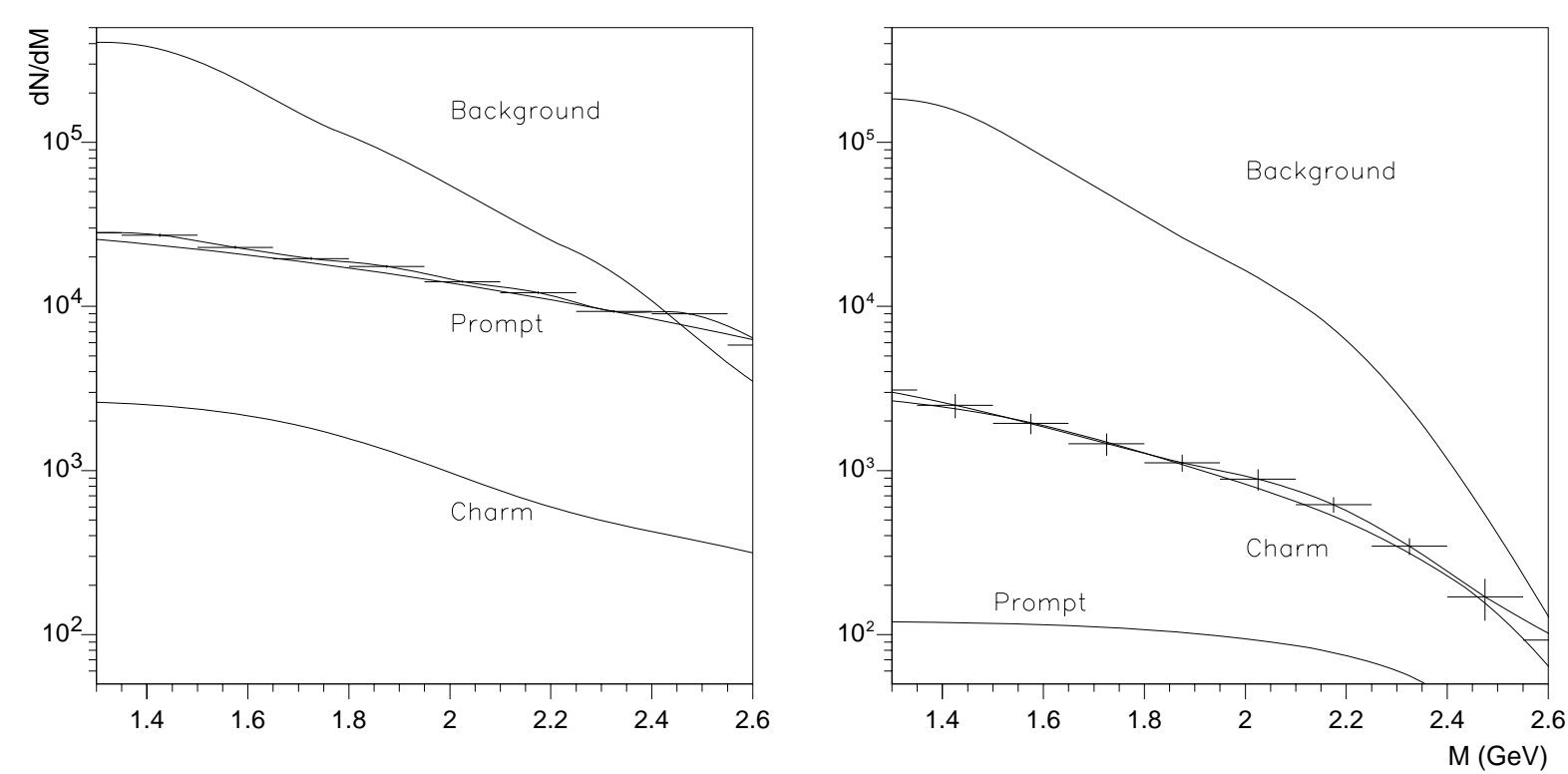

Figure 16. Simulated dimuon mass distributions for the prompt (left) and charm (right) event selections. The background contribution is also shown, including pion/kaon decays and fake matches between the tracks in the muon and in the vertex spectrometers. The error bars in the signal points include the uncertainty from background subtraction.

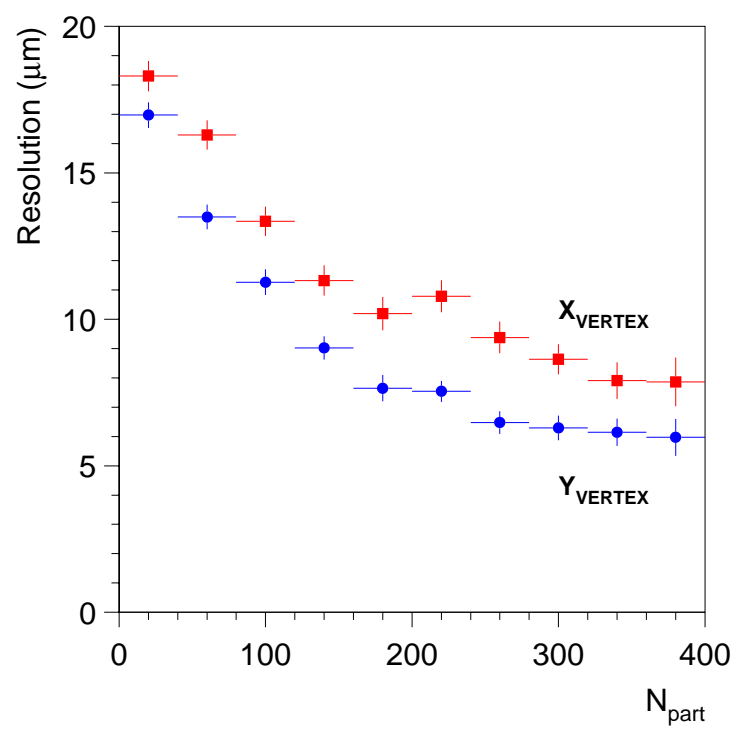

Figure 17. Calculated resolution in the determination of the transverse coordinates of the interaction vertex.

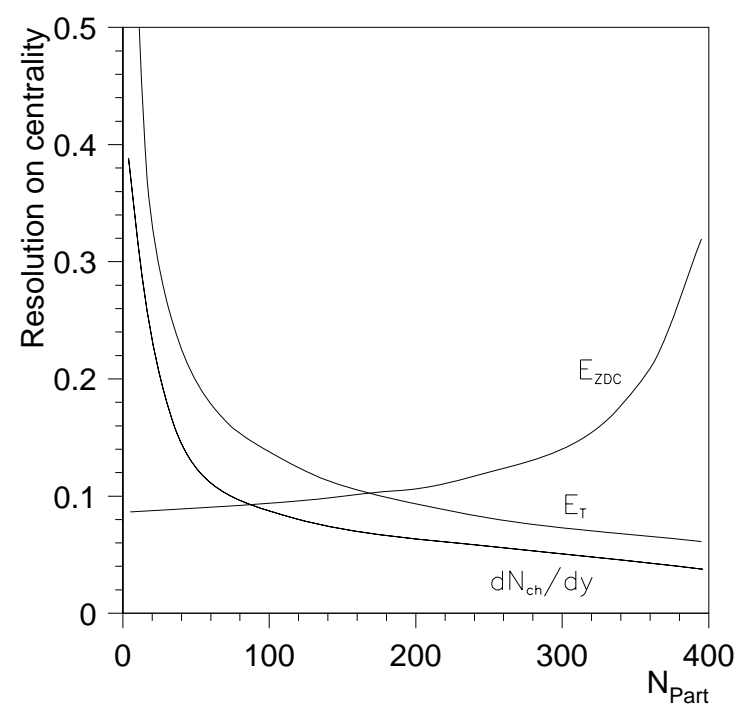

Figure 18. Resolution on the measurement of the centrality of the collisions, using three different estimators. 

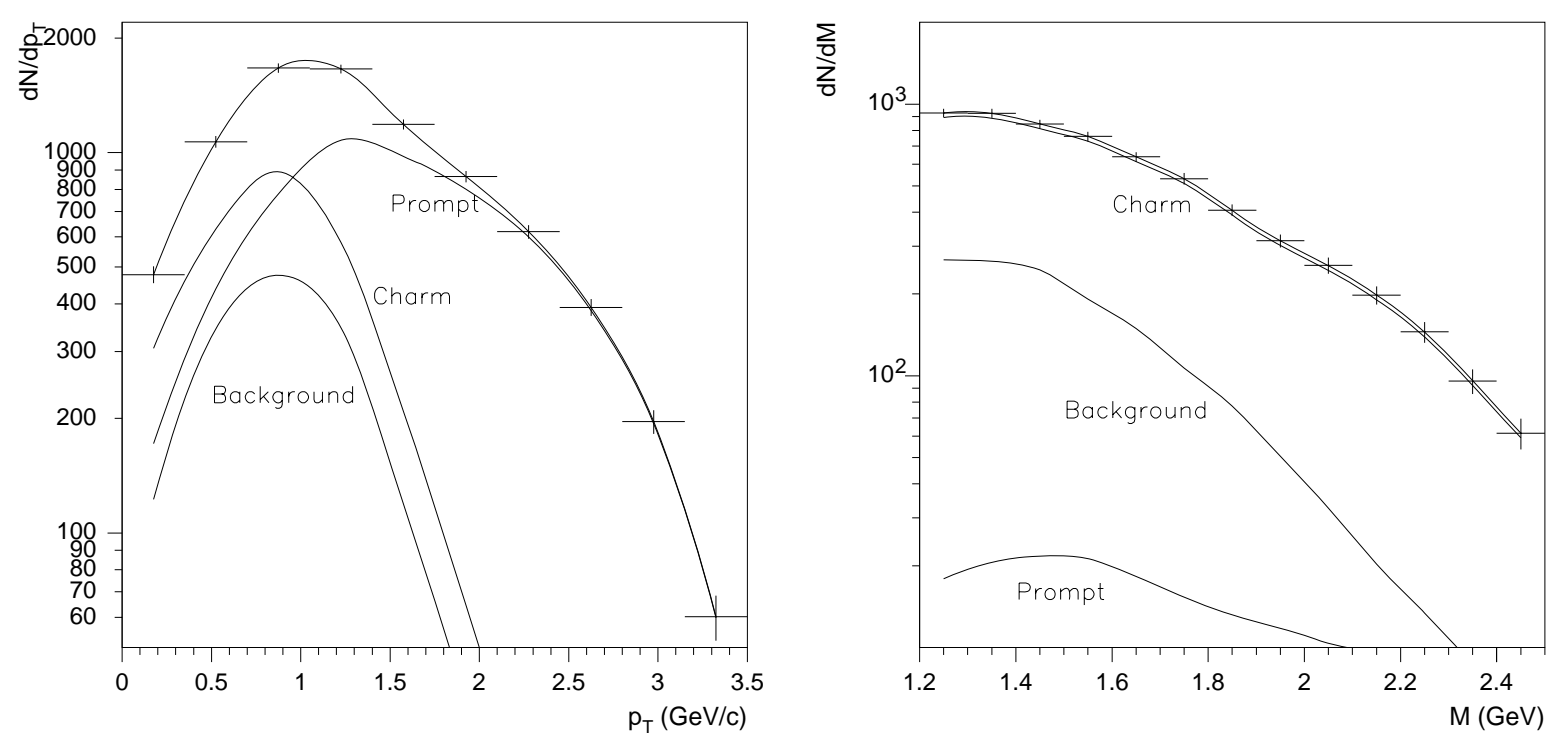

Figure 19. Expected prompt (left) and charm (right) event samples selected in proton induced collisions.

obtained with nuclear collisions. In particular, the ratio between the open charm and the Drell-Yan production cross sections will be determined with a very good accuracy in several p-A collision systems, revealing if these two hard processes have the same A-dependence or not. Figure 19 illustrates the foreseeable analysis of intermediate mass dimuons production, in $\mathrm{p}-\mathrm{Be}$ or $\mathrm{p}-\mathrm{Pb}$ collisions, showing the $p_{\mathrm{T}}$ distribution of the prompt dimuons and the mass distribution of the open charm events.

More challenging will be the measurement of the dependence of $\chi_{c}$ production on the mass number of the target, in $\mathrm{p}-\mathrm{A}$ collisions, by seeing how the ratio between $\chi_{c}$ and $\mathrm{J} / \psi$ yields changes from $\mathrm{p}-\mathrm{Be}$ to $\mathrm{p}-\mathrm{Pb}$ collisions. To minimize the systematical errors, the measurement will be done using $\mathrm{Be}$ and $\mathrm{Pb}$ targets simultaneously in the beam. The $\chi_{c} \rightarrow \psi \gamma \rightarrow \psi e^{+} e^{-}$decays will be used for this study, with the photons converting in the $\mathrm{Pb}$ target or in a $\mathrm{Pb}$ disk placed downstream of the targets, and the electron-positron pairs reconstructed in the silicon pixel telescope.

\section{SUMMARY AND CONCLUSIONS}

The results obtained since 1986 confirm that the studies of dilepton and heavy flavour production provide extremely relevant information about the (predicted) formation of a deconfined state of matter in high energy heavy ion collisions.

In view of the exciting results presently available, and of the questions remaining open, the CERN NA60 experiment was proposed, aiming at reaching a deeper understanding of the critical behaviour of QCD at SPS energies. The approval of this experiment represents an evolution from the current broad heavy ion physics programme at the SPS to a dedicated study of specific signals that have already provided very interesting results. The new measurements are expected to give a significant contribution to the understanding 
of the presently existing results, and should play a leading role in building a convincing logical case that establishes beyond reasonable doubt the formation of a deconfined state of matter in heavy ion collisions at the SPS.

\section{REFERENCES}

1. G. Agakichiev et al. (CERES Coll.), Eur. Phys. J. C4 (1998) 231.

2. G. Agakichiev et al. (CERES Coll.), Phys. Lett. B422 (1998) 405.

B. Lenkeit et al. (CERES Coll.), Nucl. Phys. A661 (1999) 23c.

3. V. Koch, Int. J. Mod. Phys. E6 (1997) 203.

4. R. Rapp and J. Wambach, hep-ph/9909229.

5. A. Marin et al. (CERES Coll.), Nucl. Phys. A661 (1999) 673c.

6. M.C. Abreu et al. (NA38/50 Colls.), Euro. Phys. J. C14 (2000) 443.

7. T. Sjostrand, Computer Physics Commun. 82 (1994) 74.

8. A.D. Martin et al., Phys. Rev. D51 (1995) 4756.

9. P. Braun-Munzinger et al., Eur. Phys. J. C1 (1998) 123.

10. M.C. Abreu et al. (NA38 Coll.), Nucl. Instrum. Meth. A405 (1998) 139.

11. C.Y. Wong and Z.Q. Wang, Phys. Lett. B367 (1996) 50.

12. Z. Lin, X.-N. Wang, Phys. Lett. B444 (1998) 245.

13. L. Capelli et al. (NA50 Coll.), in Proc. of the 4th Rencontres du Vietnam, Hanoi, Vietnam, July 2000.

14. R. Rapp and E. Shuryak, Phys. Lett. B473 (2000) 13.

15. K. Gallmeister, B. Kampfer and O.P. Pavlenko, Phys. Lett. B473 (2000) 20.

16. H. Satz, in "International School of Subnuclear Physics", A. Zichichi (Ed.), Erice, Italy, 1997, BI-TP-97-47, hep-ph/9711289.

17. J. Geiss et al., Phys. Lett. B 447 (1999) 31; J. Geiss et al., in: Proc. Workshop on Quarkonium Production in Relativistic Nuclear Collisions, Seattle, USA, May 1998 (nucl-th/9810059).

18. C. Spieles et al., Phys. Rev. C 60 (1999) 054901.

19. D.E. Kahana and S.H. Kahana, Prog. Part. Nucl. Phys. 42 (1999) 269; D.E. Kahana and S.H. Kahana, nucl-th/9908063.

20. N. Armesto, A. Capella and E.G. Ferreiro, Phys. Rev. C 59 (1999) 395.

21. D. Kharzeev, M. Nardi and H. Satz, BI-TP-97-33, July 1997, hep-ph/9707308.

M. Nardi and H. Satz, Phys. Lett. B442 (1998) 14; hep-ph/9805247.

M. Nardi, private communication, May 2000.

22. "Study of Prompt Dimuon and Charm Production with Proton and Heavy Ion Beams at the CERN SPS", A. Baldit et al. (NA60 Coll.), Proposal CERN/SPSC 2000-010, March 2000.

23. R. Shahoyan, in Proc. of the Int. Europhysics Conf. on High Energy Physics, Tampere, Finland, July 1999, p. 980.

24. K. Borer et al., Nucl. Instrum. Meth. A440 (2000) 5.

25. M. Campbell et al., IEEE Trans. on Nucl. Sci. 46-3 (1998) 156.

26. P. Sonderegger et al., Nucl. Instrum. Meth. A435 (1999) 161. 